\title{
Cambiamento di paradigma, fonti del vantaggio competitivo ed evoluzione del concetto di strategia
}

\author{
GiovanNi BATTISTA DAGNINO
}

\begin{abstract}
Obiettivo del paper: Questo contributo è teso a identificare le basi dell'evoluzione del concetto di strategia attraverso la disamina della storia evolutiva dei paradigmi dominanti negli studi di strategia d'impresa.

Metodologia: La chiave epistemologico-interpretativa prescelta è data dall'approccio evolutivo, osservato quale lente privilegiata per la comprensione dell'avvicendamento, dell'integrazione e dell'evoluzione interna dei paradigmi dominanti.

Risultati: La tesi del lavoro ruota intorno all'osservazione che il concetto di strategia è costituito da due parti fondamentali: una prima parte core, che è relativamente costante nel tempo, e una seconda parte che muta in relazione all'affermarsi delle fonti del vantaggio competitivo dei paradigmi dominanti. Sin dalle origini, la strategia d'impresa si è sviluppata secondo uno o più paradigmi dominanti che hanno agito da riferimento primario utile per illuminare il lavoro di studiosi e ricercatori. Quattro sono i paradigmi principali: (a) il paradigma Struttura-Comportamento-Performance, (b) il paradigma Risorse-CompetenzePerformance, (c) il paradigma Knowledge-Capabilities-Performance, e (d) il paradigma evolutivo. Di ognuno di essi si esaminano schematicamente struttura logica e relazioni causali, percorso evolutivo e principale contributo in relazione alla definizione di strategia.

Limiti della ricerca: Analisi esplorativa di tipo teorico-interpretativa che si potrebbe coagulare ulteriormente con osservazioni empiriche basate su interviste ad accademici, a manager e consulenti di strategia d'impresa e altresì con studi di natura bibliometrica.

Implicazioni pratiche: Si segnalano talune tendenze in tema di mutamento della teoria del vantaggio competitivo che possono avere notevoli impatto e interesse per i pratici.

Originalità del paper: Si contribuisce alla letteratura manageriale evidenziando le cause prime motivanti l'evoluzione del concetto di strategia e, più in particolare, i quattro blocchi paradigmatici alla base del cambiamento del concetto di strategia d'impresa.

Parole chiave: concetto di strategia d'impresa; paradigmi dominanti nella strategia d'impresa; cambiamento paradigmatico; epistemologia evolutiva.

Purpose of the paper: This paper aims to identify the foundations of the evolution of the concept of strategy on the ground of detecting the evolutionary history of the dominant paradigms in strategic management.
\end{abstract}

\footnotetext{
Ordinario di Economia e Gestione delle Imprese - Università degli Studi di Catania

e-mail: dagnino@unict.it
}

sinergie, rivista di studi e ricerche

n. 94, Maggio-Agosto 2014, pp. 27-50

ISSN 0393-5108 - DOI 10.7433/s94.2014.03 
Methodology: We use the evolutionary approach and evolutionary epistemology, observed as interpretative lenses effective in grasping the succession, integration and internal evolution of the dominant paradigms in strategic management.

Findings: The crux of the argument is that, the concept of strategy is composed by two fundamental parts: the core part, that is relatively abstract and permanent over time, and the peripheral part, that instead changes as relates to the sources of competitive advantage of the dominant paradigms. Actually, since its founding strategic management has developed around relevant paradigms, which have been the lighthouse for the work of researchers in the field. Four are the paradigms of strategy we present: (a) the Structure-Behavior-Performance paradigm; (b) the Resources-Competences-Performance paradigm; (c) the KnowledgeCapabilities-Performance paradigm; and (d) the evolutionary paradigm. Of each paradigm is detected its evolutionary paths, logical structure, causal relationships and specific contribution to the concept of strategy.

Research limits: Exploratory analysis of theoretical-interpretive fashion helpful to complement, with a range of empirical observations grounded in interviews to academics, executive and consultants in strategic management and with bibliometric studies.

Practical implications: We present a few intriguing trends on the change of the traditional units of analysis and the theory of competitive advantage that may have deep impact on business practice.

Originality of the paper: This paper contributes to management literature by shedding new light on the foundations underlying the evolution of the strategy concept and, in more detail, on the four key paradigms triggering the strategy concept variations.

Key words: strategy concept; dominant paradigms in strategic management; paradigm change; evolutionary epistemology

\section{Introduzione}

L'obiettivo di questo articolo è di identificare l'evoluzione del concetto di strategia d'impresa mediante l'esame del cambiamento dei paradigmi dominanti negli studi di strategia d'impresa. La tesi del lavoro gravita intorno all'osservazione che il concetto di strategia è costituito da due parti fondamentali fra loro interrelate: (a) una prima parte core, che si fa risalire alla definizione di Kenneth Andrews (1971) e che è generale, astratta e relativamente costante nel tempo; e (b) una seconda parte, più applicata e interattiva, che riguarda invece le modalità utilizzate per acquisire un vantaggio sulla competizione e muta in relazione all'affermarsi dei paradigmi dominanti. Infatti, sin dalle origini negli anni ' 60 del secolo scorso, la strategia d'impresa si è sviluppata in base a uno o più paradigmi dominanti, che hanno agito da riferimento primario utile per illuminare il lavoro di studiosi e ricercatori. Lo studio nasce dunque con l'obiettivo di proporre una lettura originale e un'interpretazione dell'evoluzione del concetto di strategia in relazione ai paradigmi dominanti dello strategic management.

Quarantatre anni or sono, Andrews (1971, p. 28) propose la definizione di strategia che segue: "Strategy is the pattern of objectives, purpose, or goals and the major policies and plans for achieving these goals, stated in such a way as to define what business the company is in or is to be in and the kind of company it is or is to 
$b e$ ". Tale definizione comprende sia gli obiettivi e i fini imprenditoriali, sia le politiche, sia anche i piani concepiti per conseguire tali obiettivi. Tuttavia si soggiunge che, nella sua generalità e quasi omnicomprensività, la definizione in parola, per dare linfa vitale al concetto di strategia, manca ancora di una qualificazione necessaria in termini delle fonti per conseguire il vantaggio competitivo. I caratteri salienti di tale seconda parte sono offerti dallo studio dei paradigmi pro-tempore dominanti. Per tale motivo, si propone la Figura 1.

Fig. 1: Le parti fondamentali del concetto di strategia

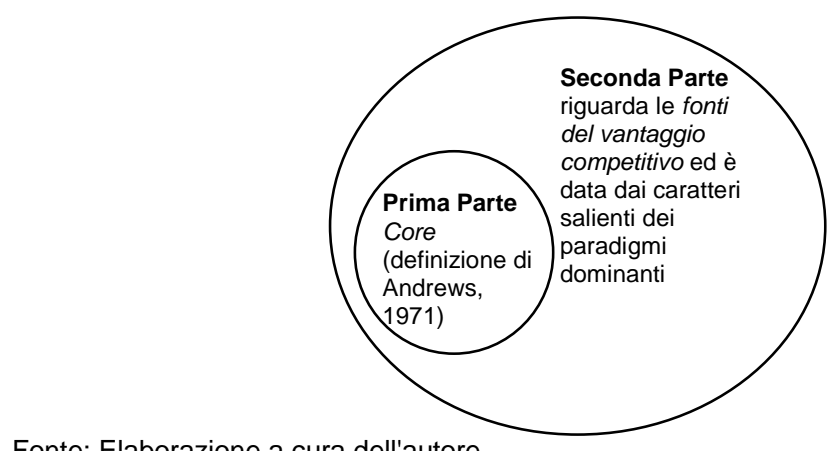

Fonte: Elaborazione a cura dell'autore

Si afferma in secondo luogo che, negli studi di strategia d'impresa, lungi dal poter parlare di una mera successione, oppure di una progressione, si ritrova invero una sorta di concatenazione e di intreccio fra paradigmi con caratteri dissimili; la qual cosa rende la loro indagine un compito complesso e problematico ma, per questa via, ancor più stimolante. La chiave prescelta è offerta dall'approccio evolutivo ${ }^{1}$, inteso nella duplice veste di chiave interpretativa dell'evolversi dei paradigmi della strategia e di paradigma evolutivo in strategia. A tal proposito, si chiarisce sin d'ora che l'approccio evolutivo, nell'accezione qui accolta, comprende la metafora biologica e la trascende estendendosi alle fondamentali interazioni sociali e relazionali nell'àmbito della comunità scientifica.

La motivazione primaria che ha condotto all'elaborazione di questo articolo è che gli studi di strategia d'impresa presentano le principali caratteristiche di una disciplina in via di maturazione a livello globale:

a) una base comune di conoscenze pressoché universalmente accettate che si polarizzano e si solidificano in alcuni textbooks (Grant, 2005; Hitt et al., 2001b;

La teoria dell'evoluzione, dal momento in cui è stata formulata da Darwin nel 1859, mai ha cessato di suscitare accesi dibattiti fra sostenitori (evolutivi) e oppositori (creazionisti e antidarwiniani in genere). Essa è adesso accettata dalla stragrande maggioranza degli studiosi e dei ricercatori nelle scienze della natura e rappresenta un punto di riferimento per tutte le discipline medico-biologiche. Tuttavia, le interpretazioni possibili sono numerose e la discussione attorno al tema dell'evoluzione è sempre vivace. 
Thompson \& Strickland, 2001; Saloner et al., 2001; Rothaermel, 2012, fra i più noti) e handbooks (Faulkner e Campbell, 2003; Hitt et al., 2001a; Pettigrew et al., 2002);

b) un linguaggio specializzato sufficientemente codificato, che serve di norma per motivi di economia di energie e di enucleazione e di identificazione disciplinare;

c) dei corsi di insegnamento specifici e dedicati di vario livello (laurea, laurea specialistica, MBA e $\mathrm{PhD}$ ) nella maggioranza delle università e business schools

d) una massa critica di ricercatori e studiosi, i quali si auto-identificano come studiosi di strategic management, e danno vita a una comunità scientifica relativamente consolidata che si riunisce in reiterate occasioni d'incontro nazionali e internazionali (fra le principali: la divisione Business Policy and Strategy dell'Academy of Management, la Strategic Management Society, la European Academy of Management - sezione di Strategic Management);

e) infine, ma non meno importante, un'estesa e articolata comunità di pratici operanti rispettivamente nelle imprese e nella consulenza manageriale che, talora parallelo, talaltra in intersezione con la precedente, diffondono la cultura strategica soprattutto nelle imprese e nei corsi di formazione anche attraverso talune riviste specializzate particolarmente influenti (si ricordano la Harvard Business Review, la MIT-Sloan Management Review e la California Management Review).

Orbene, la strategia non difetta di continuità storica. Per questo motivo, l'obiettivo primario è di descrivere "il processo di filiazione delle idee scientifiche", come lo chiama Schumpeter, ossia il processo attraverso il quale "gli sforzi degli uomini rivolti a comprendere i fenomeni economici costruiscono, migliorano e demoliscono strutture analitiche in una sequenza senza fine" (Schumpeter, 1954; tr. it., 1972, p.7). Una delle ipotesi principali di questa ricerca è che fondamentalmente tale processo non differisce da processi analoghi che si svolgono in altri campi del sapere. Eppure, per la sua relativa gioventù disciplinare, tale filiazione di idee è ancora assai recente e tutt'altro che di agevole comprensione. È sembrato pertanto opportuno provare a disegnare una storia evolutiva dei paradigmi prevalenti nel campo d'indagine e di educazione in oggetto che potesse condensare efficacemente e rielaborare in modo idoneo le conoscenze acquisite e supportare il lavoro di ricerca futuro. Si anticipa subito che si tratta di una storia di dominanze incomplete.

Il significato di paradigma qui accolto è più morbido e flessibile dell'originale nozione di Kuhn (1970) e più vicino al senso di Larry Laudan (1978) e altresì al buonsenso e alla prudenza. Con il concetto di paradigma s'intende descrivere gli standard microstorici accettati da una particolare comunità scientifica in un dato tempo storico (Ceruti, 1985; Morin, 1990). Un paradigma è dunque un tipo di relazione logica (inclusione, congiunzione, disgiunzione, esclusione) fra un certo numero di nozioni principali. In tal senso, ogni periodo storico è caratterizzato nella pratica della ricerca da un'ampia eterogeneità e da una molteplicità di idee di base coesistenti e opposte fra di loro più che da un unico affermato paradigma specifico definitivo. È questa eterogeneità che distingue, non soltanto comunità e scienziati 
differenti, ma anche le prospettive di ricerca all'interno di singola comunità e talora anche un solo ricercatore autonomo.

Secondo tale definizione, quattro sono le nozioni paradigmatiche in strategia d'impresa: (a) il paradigma Struttura-Comportamento-Performance (SCP); (b) il paradigma Risorse-Competenze-Performance (RCP); (c) il paradigma KnowledgeCapabilities-Performance (KCP); e (d) il paradigma evolutivo. Il primo, dominante negli anni '80, ha iniziato a rivelarsi non più adeguato negli anni '90 (Young, 1995), il secondo appare in fase di maturità, il terzo sembra in via di affermazione, il quarto è riuscito a distinguersi compiutamente e a trovare una sua identità (Dagnino, 2006).

Si dà conto della struttura logica del lavoro. Nel secondo paragrafo si introducono i fondamenti concettuali della prospettiva evolutiva, in particolare attraverso l'esame delle basi concettuale dell'epistemologia evolutiva di Donald Campbell e David Hull e si evidenzia il duplice ruolo della prospettiva evolutiva in strategia d'impresa. Il terzo paragrafo è dedicato all'esame delle dinamiche evolutive alla base del cambiamento paradigmatico. Applicando le premesse poste nel paragrafo precedente, il quarto paragrafo discute la dinamica evolutiva dei paradigmi dominanti in strategia d'impresa. Si evidenziano le peculiarità della sequenza evolutiva dei paradigmi della strategia. Conclude l'articolo una sezione di sintesi in cui si riprendono le fila del ragionamento, si riepilogano le principali ipotesi proposte in merito alla dinamica evolutiva degli studi di strategia d'impresa e si richiama l'attenzione sui vantaggi dell'interpretazione proposta.

\section{II duplice ruolo della prospettiva evolutiva in strategia d'impresa: chiave interpretativa del cambiamento paradigmatico e fondamento per la definizione di un paradigma evolutivo}

Nel definire le modalità con cui condurre l'interpretazione critica dei paradigmi più rilevanti per la strategia d'impresa, la prima domanda cardine è stata: è possibile identificare una chiave di lettura che consenta di sussumere la concatenazione dei paradigmi della strategia? E, nel caso affermativo, quale?

Com'è noto, individuare una chiave di lettura coerente e appropriata in tema è tutt'altro che semplice per almeno tre ordini di motivazioni. In primo luogo, il compito si rivela arduo perché, non essendo stato tentato prima, è originale almeno con riferimento alla strategia d'impresa. Esistono naturalmente alcuni account storiografici del procedere degli studi di strategia (Rumelt et al., 1994; Ghemawat, 2002), che tuttavia poco o nulla concedono a un'interpretazione analitica e di norma si risolvono in una presentazione in semplice successione, e in forma descrittiva, sintetica e stilizzata, di taluni modelli e prospettive proposti in letteratura a partire dagli anni ' 60 , non di rado con esplicite concessioni di merito generativo alla scuola harvardiana e alla scuola di Chicago (Foss, 2003).

In secondo luogo, talune espistemologie assai note e sufficientemente recenti come ad esempio la kuhniana, la lakatosiana e la laudaniana - che hanno ricevuto applicazione nelle scienze sociali e in economia, da una parte, non apparivano 
pienamente adeguate allo scopo e, dall'altra, con riferimento in particolare all'approccio di Thomas Kuhn (1970) erano già state considerate "poco" o "poco appropriate" al campo della strategia da taluni studiosi (Rumelt et al., 1994). L'approccio kuhniano tout court, in special modo, viene generalmente criticato sia per l'eccessiva indeterminatezza della nozione di paradigma (nell'opera di Kuhn, Margaret Masterman ha identificato 21 significati diversi di paradigma), sia per l'eccessivo relativismo, sia infine per l'ipersociologismo d'impostazione, ovvero per il fatto di porre enfasi spareggiata ed esclusiva sull'importanza che le comunità scientifiche (e le loro interazioni) assumono nei cambiamenti paradigmatici.

In terzo luogo, è man mano venuta alla superficie l'idea di considerare il complesso andamento della conoscenza scientifica in materia strategica come un processo di tipo evolutivo e coevolutivo, e tendenzialmente cumulativo. Così, in modo iterativo, la chiave di lettura identificata fa riferimento all'epistemologia evolutiva di Donald Campbell (1974) e David Hull (1988). Tale epistemologia, orientata com'è alla comprensione della successione nel tempo delle teorie scientifiche ovvero alla definizione di "un'epistemologia in grado di trattare evolutivamente gli allargamenti di conoscenza, le rotture dei limiti della scienza precedente, la scoperta scientifica" (Campbell, 1974; p.69), sembra di notevole aiuto nell'esame comparato dei paradigmi in strategia d'impresa. Tuttavia essa non è stata applicata in modo compiuto allo studio della strategia d'impresa.

Si riconosce immediatamente che l'epistemologia evolutiva non costituisce né l'unica strada possibile in termini metaconoscitivi e neppure la migliore in assoluto o pro tempore. Purtuttavia si ritiene che essa, corredata con le sue integrazioni e revisioni, appaia al momento la più idonea per perseguire gli scopi prefissati.

Preme evidenziare il duplice peculiare ruolo che la prospettiva evolutiva riveste in strategia d'impresa. La prospettiva evolutiva, per il suo carattere relativamente aperto e comprendente e per la sua caratterizzazione biologico-sociale (Boyd e Richerson, 1985), viene ad assumere la duplice veste di epistemologia evolutiva e di paradigma evolutivo emergente in strategia d'impresa. Questo significa che la prospettiva evolutiva contiene in sé il potenziale di proporsi, da una parte, quale base interpretativa ed epistemica per poter comprendere la sequenza evolutiva dei paradigmi della strategia, dall'altra, quale fondamento per l'elaborazione e l'affermazione di un paradigma evolutivo negli studi di strategia. In sintesi, ciò a sua volta significa che la prospettiva evolutiva è alla base dell'evoluzione del flusso di conoscenze in strategia d'impresa; conoscenze che possono essere 'potenziate', 'velocizzate' e 'accelerate' qualora si applichi la prospettiva evolutiva.

Orbene, questo singolare duplice ruolo che la prospettiva evolutiva può rivestire viene evidenziato da tre caratteristiche fondamentali che sono di seguito esaminate:

a) l'enhancement evolutivo (o arricchimento cognitivo, epistemico e sociorelazionale);

b) la non neutralità evolutiva;

c) la possibilità di ricomprendere e conciliare micro e macro processi evolutivi. 


\subsection{L'enhancement evolutivo}

Per quanto concerne il concetto di enhancement evolutivo, si fa riferimento al ragguardevole potenziale di arricchimento e di velocità, e dunque di incremento dell'adattamento e del fitness (o idoneità) inclusivo, che può derivare dall'applicazione della prospettiva evolutiva alla strategia d'impresa. Anzitutto, l'approccio evolutivo agisce a diversi livelli del campo manageriale, livelli che sono sia teorici sia metateorici. Così, a livello metateorico, l'enhancement consente di comprendere meglio la genesi e lo sviluppo dei paradigmi, le interrelazioni, le convergenze e le divergenze, le integrazioni (reali o potenziali), le filiazioni e le speciazioni paradigmatiche che sono solite avvenire anche per le interrelazioni sociali fra studiosi, fra gruppi di ricerca e nelle e fra le comunità scientifiche. L'enhancement evolutivo è reso di norma possibile dai processi coevolutivi, ricorsivi e autoalimentanti cosiddetti di feedforward.

Tali relazioni metateoriche di potenziamento della coerenza si verificano a un livello fondamentale: si tratta di relazioni intraparadigmatiche, quando queste avvengono sostanzialmente nell'àmbito delle teorie di un paradigma; si tratta invece di relazioni interparadigmatiche, quando esse sono identificabili quali relazioni esterne al singolo paradigma, ovvero relazioni di arricchimento fra paradigmi differenti. Ancora, l'enhancement evolutivo può giovare a livello teorico, dal momento che pone le basi per studiare e comprendere meglio, da una parte, significato, ruolo e campo di applicazione delle singole teorie che vengono via via utilizzate (teoria della conoscenza, teoria delle risorse, e così via) e, dall'altra, natura, potenzialità e velocità evolutive delle varie categorie e dei vari livelli analitici (e delle interrelazioni fra questi) che di norma formano oggetto di analisi in strategia d'imprese (imprese singole, reti e popolazioni e sistemi di imprese, settori, gruppi di individui e singoli individui).

\subsection{La non neutralità evolutiva}

Riguardo alla peculiare proprietà evolutiva di non neutralità, ci si imbatte in una proprietà sostanzialmente speculare a quella relativa all'enhancement e, proprio per questo di grandissimo interesse ai fini di questo studio: il che avviene perché si fa riferimento specifico all'assenza di neutralità adattiva complessiva della prospettiva evolutiva. Com'è noto, quando si è alla presenza di neutralità, non si manifestano differenze apprezzabili nella fitness inclusiva degli individui che fanno parte di una popolazione. In questa situazione, le differenti variazioni che pure via via emergono non aggiungono né tolgono alcunché allo stato di adattamento di un individuo; esse sono dunque 'neutrali' rispetto all'idoneità relativa di un individuo di una specie.

Giacché l'applicazione della prospettiva evolutiva alla strategia d'impresa condiziona in modo significativo sia il grado di idoneità adattiva relativa delle teorie e dei paradigmi sia lo sviluppo di un paradigma evolutivo, perché è in grado di modificare significativamente $\mathrm{i}$ rispettivi percorsi evolutivi degli elementi in discorso, possiamo affermare che essa presenta proprietà di non neutralità. Ciò 
naturalmente è lungi dal significare che, nel corso degli sviluppi (meta)teorici in strategia d'impresa o anche nella storia di un paradigma evolutivo, non vi possano essere periodi di stasi o neutrali anche in virtù di singole o di una serie di mutazioni neutrali. Tuttavia, nel complesso, la prospettiva evolutiva rifugge di norma da applicazioni neutrali, o eccessivamente conservatrici e unilineari, presentando invece taluni singolari e imprescindibili caratteri di effettiva azione trasformatrice e acceleratrice che può favorire l'ottenimento di risultati non intenzionali in presenza di azioni del tutto intenzionali (Hayek, 1945; 1988; Merton, 2002), estendendo lo spazio d'incertezza e d'indeterminatezza ex ante del periodo futuro.

\subsection{La possibilità di ricomprendere e collegare micro e macro processi di evoluzione}

La prospettiva evolutiva, per il suo carattere inclusivo e coevolutivo, offre la possibilità di ricomprendere e di collegare in modo armonico micro processi evolutivi, che sono soliti verificarsi a livello d'impresa singola o nell'ambito della medesima, e macro processi evolutivi, che sono soliti realizzarsi a livello di sistemi di imprese e di sistema economico. In tal modo, la prospettiva evolutiva ricomprende i processi e le relazioni che avvengono al mesolivello, ossia le relazioni intersistemiche fra imprese che fanno parte di sistemi di imprese (Dagnino, 2000) e le relazioni fra sottosistemi nell'ambito della singola impresa. Questa importantissima potenzialità di riconciliazione tramite la coevoluzione dei processi micro-macro richiama a sua volta tangibilmente sia il comportamento micro-macro, un ramo di fatto ancor quasi vergine nella letteratura economica, sia ancora la coevoluzione dei caratteri dinamici d'industria e d'impresa, ossia la competitive organizational behavior (Barney e Zajac, 1994) in strategia d'impresa.

\section{Le dinamiche evolutive del cambiamento paradigmatico}

Posto che la prospettiva evolutiva riveste un duplice essenziale ruolo in strategia d'impresa, ovvero cardine epistemologico interpretativo dell'avvicendamento e della sequenza evolutiva dei paradigmi dominanti e fondamento per la proposizione di un paradigma evolutivo, in questo paragrafo si affronta in qual modo e in qual misura la prospettiva evolutiva può essere di sostegno utile nell'interpretazione dell'avvicendamento dei paradigmi della strategia, e quindi nella mappatura delle sequenze evolutive, delle integrazioni evolutive o fusioni, delle evoluzioni divergenti o speciazioni, delle coevoluzioni fra differenti paradigmi.

Si è dunque pensato di studiare i driver del cambiamento dei paradigmi della strategia. Occorre a tal fine esaminare le modalità con cui la prospettiva evolutiva può servire da guida nella ricostruzione delle sequenze, delle integrazioni, delle evoluzioni divergenti o speciazioni, delle coevoluzioni fra differenti paradigmi in strategia d'impresa. Si tratta di una prospettiva che ha carattere più proscrittivo che prescrittivo, di una sorta di bussola per la navigazione notturna in acque ignote o 
poco conosciute, che indica vari percorsi possibili e praticabili, giacché essa può indicare cosa accade o può accadere, ma non deve necessariamente accadere, nella dinamica fra paradigmi in strategia d'impresa.

Nella Figura 2 di cui alla pagina successiva è contenuta una serie di interazioni paradigmatiche tipiche che danno la misura dei risultati ottenibili dal cambiamento evolutivo attraverso le dinamiche interparadigmatiche possibili. Esse vengono esaminate di seguito:

(i) la sequenza evolutiva del paradigma A;

(ii) l'evoluzione divergente o speciazione del paradigma A;

(iii) l'integrazione evolutiva o fusione dei paradigmi A e B;

(iv) la coevoluzione dei paradigmi A e B.

\section{(i) Sequenza evolutiva dei paradigmi}

Per quanto riguarda la sequenza evolutiva tipica del paradigma A, contenuta nella parte "A" della Figura 2, si tratta di un modello di base che spiega analiticamente l'evoluzione temporale del paradigma A, che avviene di solito per replicazione, come cambiamento sequenziale entro il sistema: una parte (o più) dello spazio concettuale del paradigma $\mathrm{A}$ al tempo $\mathrm{t}_{1}$, che si può chiamare ad esempio $\mathrm{x}$, viene trasformata al tempo $t_{2}$ nella forma variante $y$, e ancora nella variante $\mathrm{z}$ al tempo $t_{3}$, con $\mathrm{x}, \mathrm{y}, \mathrm{e} \mathrm{z}$ che differiscono per il loro contenuto informativo. In pratica, ciò significa che un determinato paradigma evolve in modo semiconservativo per variazione di una o più delle sue parti, in modo tale che - a distanza di alcuni periodi - esso può assumere forme anche assai diverse da quella iniziale o fondativa. Naturalmente, la sequenza evolutiva di un paradigma può originare tanto endogenamente quanto esogenamente, ossia le mutazioni paradigmatiche possono avere talora natura interna (a motivo delle scelte degli studiosi, presi ora come singoli, ora come successive generazioni), oppure talaltra essere indotte dall'esterno sotto lo stimolo di paradigmi o teorie alternative.

\section{(ii) Evoluzione divergente o speciazione di un paradigma}

Nella parte "B" della Figura 2 viene mostrata la speciazione paradigmatica o evoluzione divergente, ossia la filiazione o suddivisione del paradigma $\mathrm{A}$ in due o più paradigmi-figli. Si tratta di una ramificazione per differenziazione paradigmatica secondo la quale, dalla partizione di un unico paradigma $\mathrm{A}$ si ottengono due o più paradigmi diversi (nel caso che viene suggerito il paradigma A e il paradigma B), che mostrano contenuti in parte differenti fra loro. Di norma, almeno nelle fasi iniziali post-speciazione, nel paradigma B si ritrova una parte comune ad A e una parte caratterizzata da una novella variazione che si enuclea e si manifesta per differenza rispetto ad A e viene definita zona o regione di differenza (Durham, 1991; p. 184), identificata in Figura 2 con la lettera ' $H$ '. Di norma dunque, al di fuori della regione di differenza, i due paradigmi-figli presentano una sostanziale unità tipologica prodotta dalla comune discendenza. Per motivi illustrativi, i confini complessivi dello spazio concettuale dei paradigmi vengono mostrati eguali e costanti in forma e dimensione, mentre esse in realtà essi variano di caso in caso, 
tanto nell'àmbito di un determinato paradigma quanto nel tempo, dando vita talora a regioni evolutive gerarchicamente complesse e articolate.

Fig. 2: Dinamiche di evoluzione e di coevoluzione dei paradigmi in strategia d'impresa

(A) Sequenza evolutiva temporale tipica del paradigma $\mathrm{A}$

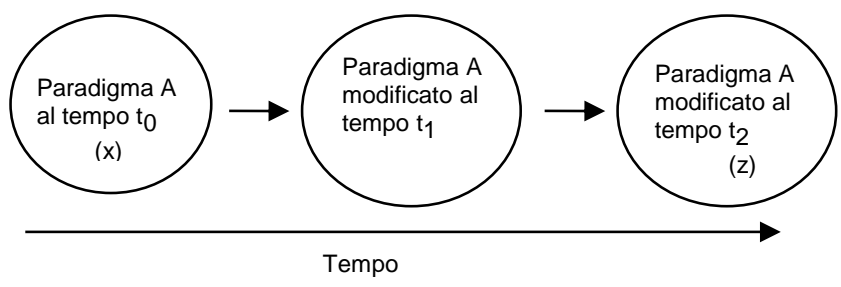

(B) Evoluzione divergente (o speciazione o filiazione) del paradigma A

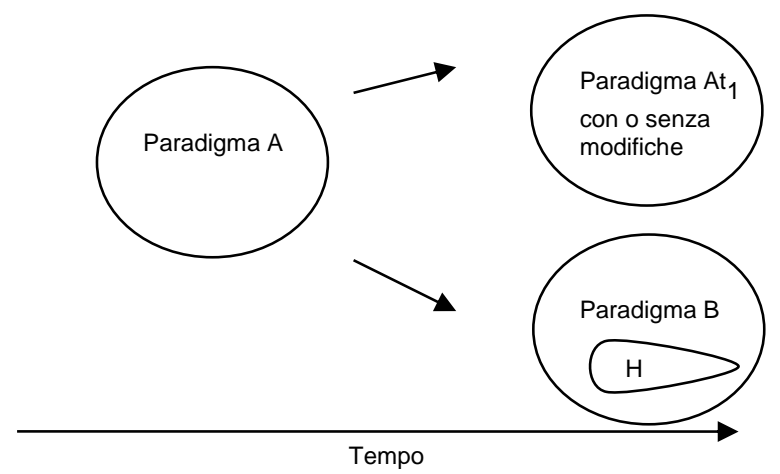

(C) Integrazione o fusione evolutiva dei paradigmi A e B

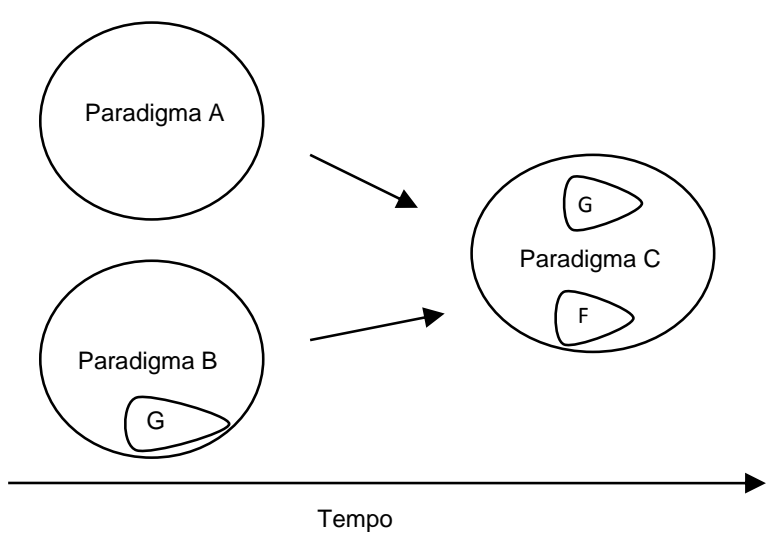




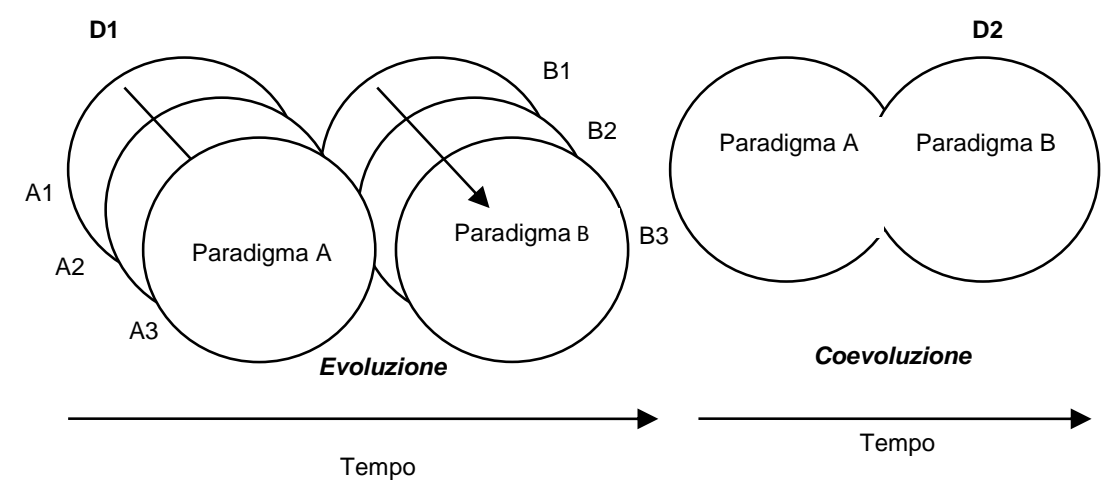

Fonte: Elaborazione a cura dell'autore

(iii) Integrazione evolutiva o fusione fra paradigmi

La parte "C" della Figura 2 presenta l'integrazione evolutiva fra paradigmi; si tratta di un modello che comprende la circostanza che due (o più) paradigmi originari A e B si fertilizzino a vicenda sino a intersecarsi fra di loro e a fondersi in modo tale da dar vita a un terzo paradigma C. È questa la forma che in natura assume una tipica riproduzione sessuata che, a propria volta, permette il rimescolamento dei cromosomi, il continuo riassorbimento delle forme presenti in una popolazione, la fusione dei nuclei e la formazione di combinazioni genetiche sempre nuove. Specularmente, rispetto al modello della speciazione in cui si erano identificate delle zone/regioni di differenza, lo spazio concettuale del paradigma effetto dell'integrazione evolutiva mostra delle zone o regioni di identità (alle lettere ' $F$ ' e ' $G$ ' in Figura 2 parte $C$ ), che erano già presenti nei paradigmi originari $A$ e $B$ e sono state replicate (sebbene non di rado non senza variazioni e senza la medesima combinazione) nel paradigma-risultato C. D'altra parte nel caso dell'integrazione interparadigmatica è quasi sempre possibile osservare (e ricostruire ex post) il processo di crossing-over, che comporta lo scambio di materiale genetico fra i due paradigmi, e suole avvenire per le interazioni sociali fra studiosi e ricercatori provenienti dai paradigmi originari.

(iv) Coevoluzione di due o più paradigmi

Infine, la dinamica coevolutiva fra paradigmi A e B illustrata nella parte "D" della Figura 2. Questa singolare dinamica interattiva fra due (o talora più) paradigmi base si esprime attraverso l'accoppiamento delle sequenze evolutive dei paradigmi originari A e B (si veda D1), l'evoluzione simultanea (di forma e contenuti) di tali paradigmi e la loro codeterminazione evolutiva, dando luogo a risultati non prevedibili e talora sorprendenti. La codeterminazione evolutiva fra paradigmi, ovvero la (ri)combinazione di diversi sentieri evolutivi di eredità informativa, è 
ottenuta attraverso l'auto-organizzazione da parte degli studiosi provenienti da diverse comunità scientifiche base e con la mediazione dei processi autoalimentanti di retroazione positiva (o feedforward) prima esaminati, che accelerano, potenziano e rendono più agevole e vantaggiosa l'interazione e lo scambio interparadigmatici. Tali processi mettono di norma in moto una serie di interazioni ricorsive e circolarità causali inter e intraparadigmatiche con itinerari ed esiti incerti. Le interazioni coevolutive fra paradigmi si trovano dunque all'origine dell'emersione di diversità, variazioni e innovazioni euristiche e paradigmatiche che sono impredicibili a priori e possono assumere vasta portata nella dinamica multiparadigmatica.

\section{L'evoluzione dei paradigmi competitivi in strategia d'impresa}

Si ricostruisce in questo paragrafo la sequenza di evoluzione dei paradigmi competitivi in strategia d'impresa, muovendo dal primo paradigma consolidato e dominante negli anni '80, il paradigma SCP, esaminando dunque i paradigmi RCP e KCP affermatisi negli anni '90 del secolo scorso, sino a giungere al paradigma evolutivo in via di definitiva affermazione.

\subsection{La storia evolutiva dei paradigmi della strategia d'impresa come storia di dominanze incomplete}

È forse utile richiamare che la storia evolutiva dei paradigmi in strategia d'impresa è, sin dalle origini, una storia di dominanze incomplete nello spazio strategico evolutivo ${ }^{2}$, ovvero di dominanze imperfette, tendenzialmente non esclusive, mai definitive. Si direbbe che si tratta di una circostanza fortunata, perché questa condizione - pressoché permanente nel campo della strategia - ha consentito una certa effervescenza intellettuale, la lievitazione naturale degli argomenti più rilevanti e delle prospettive più significative, e una progressiva articolazione paradigmatica di mano in mano che avveniva l'emersione dei nuovi approcci e delle nuove modalità esplicative.

Questa condizione si è tradotta in realtà per due principali motivi: anzitutto i paradigmi della strategia, ora relativamente maturi, ora in via di maturazione, ora

2 Per spazio strategico evolutivo s'intende l'area concettuale nel quale si svolgono le sequenze evolutive e le interazioni fra paradigmi della strategia d'impresa. Ogni qualvolta uno studioso utilizza, anche in sede di test empirico, una specifica teoria che fa parte di un paradigma, egli agisce per replicare e/o modificare, più o meno consapevolmente, una parte dello spazio strategico. Allo spazio strategico evolutivo possono essere apportate modifiche che, nel tempo, possono rivelarsi non poco rilevanti senza intenzionalità da parte del ricercatore. Naturalmente quando avvengono tramite relazioni interparadigmatiche e interteoriche guidate, le variazioni e le mutazioni che lo spazio strategico riceve a opera degli studiosi sono del tutto intenzionali. Eppure, malgrado l'intenzionalità, i risultati complessivi ottenuti in modo evolutivo e cumulativo possono essere del tutto diversi da quelli inizialmente attesi. 
emergenti o in via di solidificazione, sono dei paradigmi parziali, incompleti e imperfetti, che non forniscono spiegazioni esaustive e definitive alle problematiche delle imprese. In secondo luogo, nessun paradigma via via dominante o codominante ( ia esso il paradigma SCP o il paradigma RCP) ha potuto sinora contare su di un dominio assoluto e incontrastato nello spazio strategico evolutivo, lasciando asilo a voci dissenzienti e a possibili fertilizzazioni originate per vie esterne rispetto alla prospettiva dominante. La qual cosa altro non è che il sintomo più evidente del polimorfismo e della effervescenza concettuale ed epistemica degli studi di strategia d'impresa che, anche per la loro origine multidisciplinare, mai ha albergato - se si eccettua verosimilmente una parte degli anni ' 80 in relazione al paradigma SCP - un unico approccio paradigmatico in modo pressoché assoluto e incontrastato nello spazio strategico evolutivo.

Posta questa premessa, utile a riportare nella giusta luce e nella giusta prospettiva la forza e l'efficacia relative dei paradigmi competitivi, si esaminano gli andamenti paradigmatici che danno vita a una sequenza evolutiva unica e originale della strategia d'impresa. Si affida a studi dedicati l'esame particolare delle articolazioni dei paradigmi oggetto di studio (Dagnino, 2005).

\subsection{La sequenza evolutiva dei paradigmi in strategia d'impresa}

La conoscenza riferita all'impresa nell'incessante ricerca della natura e delle fonti del vantaggio competitivo ha presentato, nelle ultime tre decadi, sia un rilevante arricchimento concettuale sia un progressivo ispessimento teorico: dapprima - come si osserva in Figura 3.A - l'ascesa e l'affermazione del paradigma SCP, che pone specifica attenzione sui fattori strutturali esterni alle imprese, e successivamente l'emergere e la dominanza parziale del paradigma $\mathrm{RCP}$, che è invece orientato ai fattori endogeni delle imprese, ovvero alle risorse e alle competenze che queste si procurano e sviluppano (di cui alla Figura 3.B). Più recentemente, sono emersi e in via di affermazione, accanto alle precedenti, altre due rilevanti prospettive paradigmatiche: il paradigma $\mathrm{KCP}$, originato in parte quale speciazione del paradigma RCP, che è basato sulla protezione-creazione della conoscenza a livello d'impresa; e il paradigma evolutivo, fondato sulla prospettiva biologica darwiniana, e orientato alla costruzione, alla manutenzione e al cambiamento delle routine organizzative (in Figura 3.C).

Coerentemente, la sequenza evolutiva dei paradigmi della strategia d'impresa sembra quindi mostrare un duplice passaggio evolutivo:

(a) il primo passaggio, da un orientamento che pone l'enfasi sui fattori esogeni o ambientali a una prospettiva che guarda ai fattori più squisitamente endogeni delle imprese, siano essi tangibili o intangibili o legati ai flussi di conoscenza (Hoskisson et al., 1999);

(b) il secondo passaggio, da un chiaro orientamento endogeno alle risorse e alla conoscenza a una (o meglio più) schematizzazioni tendenzialmente dinamiche, processuali e coevolutive (talora ibride e in via di definizione) che si richiamano all'operare del paradigma evolutivo (Dagnino, 2006). 
Fig. 3: Sequenza delle dominanze incomplete dei paradigmi nello spazio strategico evolutivo

(A) Anni '80

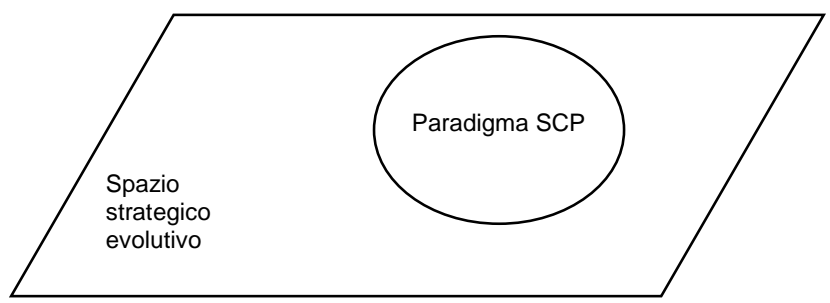

(B) Anni '90

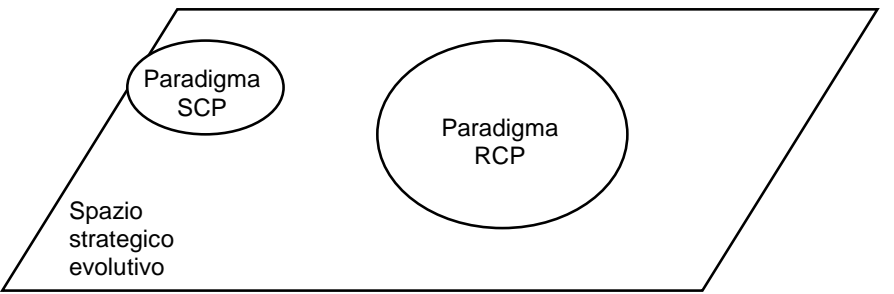

(C) Seconda metà anni '90 e primo quindicennio del 2000

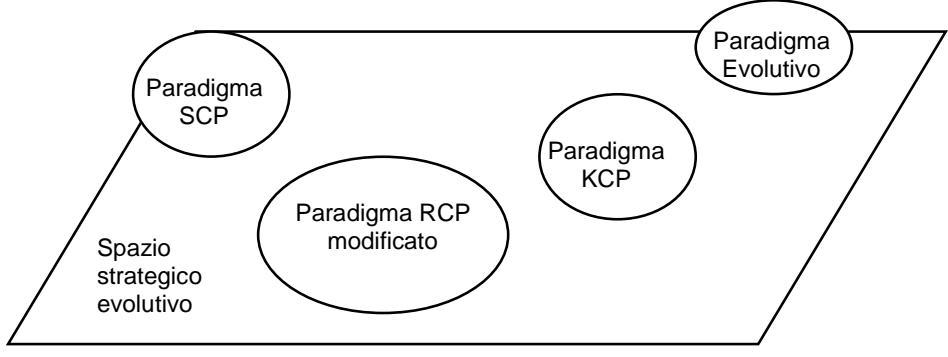

Fonte: Elaborazione a cura dell'autore

Tab. 1: Sequenza evolutiva dei paradigmi in strategia d'impresa

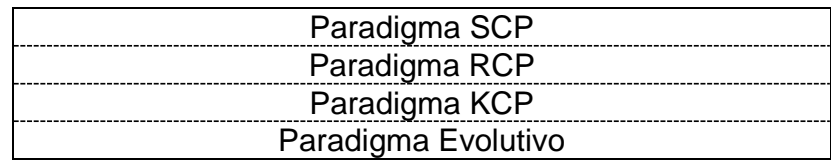

Fonte: Elaborazione a cura dell'autore

Da qui l'esigenza di uno studio sulla natura e sullo svolgimento delle due fasi del processo evolutivo, del duplice passaggio teorico-concettuale e della loro applicazione. 


\subsection{II paradigma SCP}

Come si osserva in Figura 4.A, nella strategia d'impresa durante gli anni ' 80 si rivela centrale il modello competitivo radicato nel paradigma economico industriale Struttura-Comportamento-Performance (Scherer e Ross, 1990; Porter, 1980; 1981), che convalida l'analisi della struttura industriale quale mezzo fondamentale per valutare il potenziale competitivo delle imprese del settore. In questo periodo, il paradigma SCP evolve sostanzialmente in modo endogeno e determinista passando per la priorità assoluta accordata alla struttura di settore (approccio strutturalista) e per un ruolo iniziale assegnato al comportamento delle imprese (approccio comportamentista). I modelli economico-industriali di derivazione nordamericana guardano essenzialmente al di fuori delle imprese per spiegare risultati 'continuativamente' superiori alla media, esaminando varie strutture di mercato, configurazioni pubbliche di controllo alternative, relazioni collusive, o tecnologie sostitutive. Si parla in tal caso di approccio strutturalista del paradigma SCP. Sin dai tardi anni ' 70 , una seconda prospettiva, quella europea (Jacquemin e De Jong, 1977), al paradigma SCP aveva invero posto attenzione sul comportamento delle imprese, dando vita di fatto all'approccio comportamentista al paradigma in discorso. Tale approccio pone maggior enfasi sul ruolo attivo delle strategie delle imprese operanti nel settore (o potenziali entranti), che possono avere un notevole impatto sulle sue performance e sulla struttura del settore di riferimento (paradigma $\mathrm{SCP}$ modificato al tempo $\mathrm{t} 1$ ). Si ha infine una terza interpretazione più recente e di tipo maggiormente interattivo fra le varie parti che costituiscono il paradigma, ovvero struttura di settore, comportamento delle imprese e loro performance (paradigma SCP modificato al tempo t2). In ogni modo, secondo tale approccio paradigmatico le fonti del vantaggio competitivo dell'impresa fanno sostanziale riferimento al posizionamento relativo dell'impresa medesima (e/o del business) nel settore di riferimento. Tale posizionamento è considerato la chiave di volta dell'intero processo strategico.

Fig. 4a: Dinamiche di evoluzione e di coevoluzione dei paradigmi della strategia d'impresa

\section{(A) Sequenza evolutiva del paradigma SCP in strategia d'impresa}

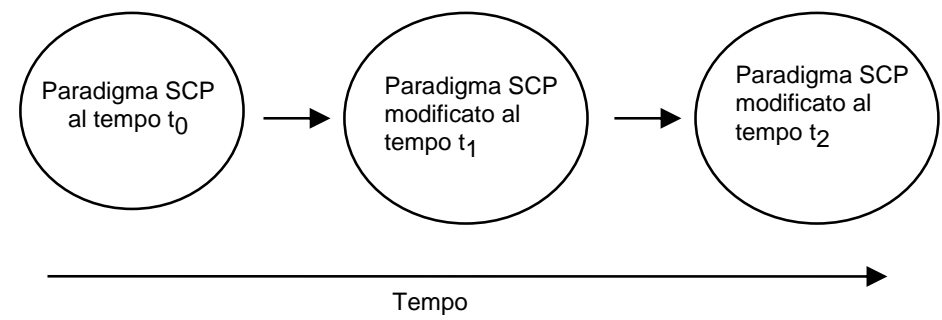

Fonte: Elaborazione a cura dell'autore 


\subsection{II paradigma $R C P$}

Posto che un numero elevato di contributi ha ripetutamente messo in dubbio l'influente punto di vista dell'Industrial Organization Economics (Rumelt, 1991; Baden-Füller e Stopford, 1994), nel corso degli anni '90 l'interesse principale degli studiosi si è progressivamente rivolto prima, agli inizi della decade, alla teoria dell'impresa basata sulle risorse (resource-based) e poi, nella seconda metà del decennio, anche alla teoria dell'impresa fondata sulla conoscenza (knowledgebased). Questa condizione è avvenuta almeno parzialmente quale reazione all'intenso e duraturo influsso dell'economia industriale nel pensiero strategico, e forse ancor più a causa delle differenti configurazioni socio-economiche $\mathrm{e}$ tecnologiche dei mercati e delle imprese nei processi di globalizzazione (si veda la Figura 4 alle lettere B e C). Secondo tali teorie dell'impresa, la fonte delle performance delle imprese è essenzialmente interna o endogena all'impresa medesima: si può così parlare, rispettivamente, del paradigma RCP (RisorseCompetenze-Performance) e del paradigma KCP (Knowledge-CapabilitiesPerformance). Per i paradigmi RCP e KCP, le differenze persistenti nelle prestazioni delle imprese e nelle rendite economiche da essi prodotte vengono guidate e dirette principalmente dalle risorse, dalle capacità strategiche e dalle conoscenze di queste, e non invece dalle loro strategie di posizionamento di prodotto e di mercato o dalle caratteristiche strutturali dei settori industriali nei quali esse si trovano a operare (ossia dall'ambiente competitivo di matrice porteriana). La fonte del vantaggio strategico risiede, in ultima analisi, nel possesso e nei processi di allocazione, rispettivamente, delle risorse, delle capacità e delle conoscenze.

Il paradigma RCP ha seguito in sostanza un percorso evolutivo divergente speciando in due differenti scuole di pensiero relativamente distinte (Schultze, 1994): il paradigma $\mathrm{RCP}_{1}$ o paradigma 'iniziale' e 'statico' delle risorse (Barney, 1991, Peteraf, 1993), da una parte, e il paradigma $\mathrm{RCP}_{2}$ o 'processuale' e delle 'capacità dinamiche' (Teece et al., 1997; Eisenhardt e Martin, 2000), dall'altra (si veda la Figura 4.B).

Fig. 4b: Dinamiche di evoluzione e di coevoluzione dei paradigmi della strategia d'impresa

(B) Evoluzione divergente e successiva integrazione evolutiva del paradigma RCP

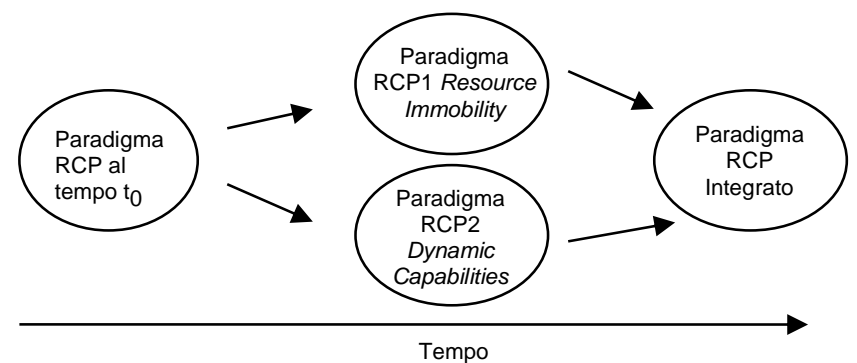

Fonte: Elaborazione a cura dell'autore 
Com'è possibile osservare, i due paradigmi RCP sono il frutto di una singolare e irreversibile speciazione evolutiva e si distinguono fra loro per la provenienza delle rendite e per le condizioni e i processi, secondo cui le differenti tipologie di rendite possono venire considerate competitivamente importanti: in estrema sintesi l'appropriazione di valore nel caso del paradigma $\mathrm{RCP}_{1}$ e la creazione di valore in relazione alla $\mathrm{RCP}_{2}$. Più recentemente, si è invece assistito alla convergenza e alla (ri)combinazione delle due 'scuole delle risorse' verso il ricongiungimento e l'integrazione evolutiva.

\subsection{II paradigma KCP}

Principiato verso la metà degli anni ' 90 come evoluzione divergente del paradigma RCP, l'andamento della prospettiva basata sulla conoscenza è stato complesso e stimolante (Eisenhardt e Santos, 2002), e ha fatto registrare, dapprima una speciazione (da RCP a $\mathrm{KCP}_{1}$ ), e poi una sequenza evolutiva svoltasi pressoché in parallelo alla prima (dalla teoria nonakiana della creazione di conoscenza all'impresa quale soggetto che crea continuamente conoscenza). È così possibile introdurre l'ipotesi dell'esistenza, ma per processi evolutivi paralleli di speciazione e di adattamento, di un duplice paradigma KCP: il paradigma $\mathrm{KCP}_{1}$ e il paradigma $\mathrm{KCP}_{2}$ (Figura 4.C). Il paradigma KCP è rimasto almeno inizialmente connesso saldamente all'appropriazione di valore, considerando l'impresa un soggetto che sviluppa superiori capacità di protezione (Porter-Liebeskind, 1996) e di integrazione (Grant, 1996) della conoscenza: si può così parlare di $\mathrm{KCP}_{1}$.

Con l'emersione del paradigma $\mathrm{KCP}_{2}$, l'impresa viene osservata piuttosto quale soggetto per la produzione di conoscenza, o anzi come entità che crea conoscenza (Nonaka et al., 2000; Nonaka e Toyama, 2002): la conoscenza e la capacità di creare e di utilizzare questa medesima conoscenza costituiscono le fonti più importanti del vantaggio competitivo.

\subsection{II paradigma evolutivo}

Parallelamente all'emergere del paradigma $\mathrm{KCP} 2$, ovvero la versione tesa alla creazione di conoscenza e valore innovativo, si assiste alla formazione di un ulteriore paradigma in strategia: il paradigma evolutivo, così detto perché radicato, tanto nella prospettiva economico evolutiva (Nelson e Winter, 1982) e nell'approccio economico-organizzativo comportamentista che fa tradizionalmente capo alla cosiddetta "Carnegie School" (March e Simon, 1958; Cyert e March, 1963; Gavetti, Levinthal e Occasio, 2007). Tale paradigma, per sua natura prevalentemente valutativo, dinamico e processuale, è andato enucleandosi in modo tale da assumere una precisa identità che, a livello di percorsi di sviluppo d'impresa, è legata al plasmarsi dei concetti di competenza, di apprendimento e di routine (Nelson e Winter, 2002). Per tale motivo, la fonte del vantaggio competitivo dimora in questo paradigma nelle routine e nei processi di innovazione e di apprendimento strategico, 
piuttosto che nel posizionamento nel settore o nelle risorse, capacità e conoscenze (Baum e Singh, 1994; Barnett e Burgelman, 1996), come si vede nella Figura 4E. È utile soggiungere che il paradigma evolutivo è per sua natura un paradigma assai meno deterministico e più aperto e permeabile, con una razionalità organica più debole e comprendente dei paradigmi SCP, RCP, KCP. Per tali motivi, esso è un paradigma potenzialmente coevolutivo che può accomodare, anche dal punto di vista metodologico ed epistemologico, diverse tradizioni in ambito strategico, fungere da momento integrativo e spingere l'evoluzione concettuale ed epistemica e l'enhancement evolutivo delle medesime (Nelson e Winter, 2002).

Fig. 4c: Dinamiche di evoluzione e di coevoluzione dei paradigmi della strategia d'impresa

(C) Dinamiche evolutive e di interscambio fra i paradigmi RCP, KCP ed evolutivo

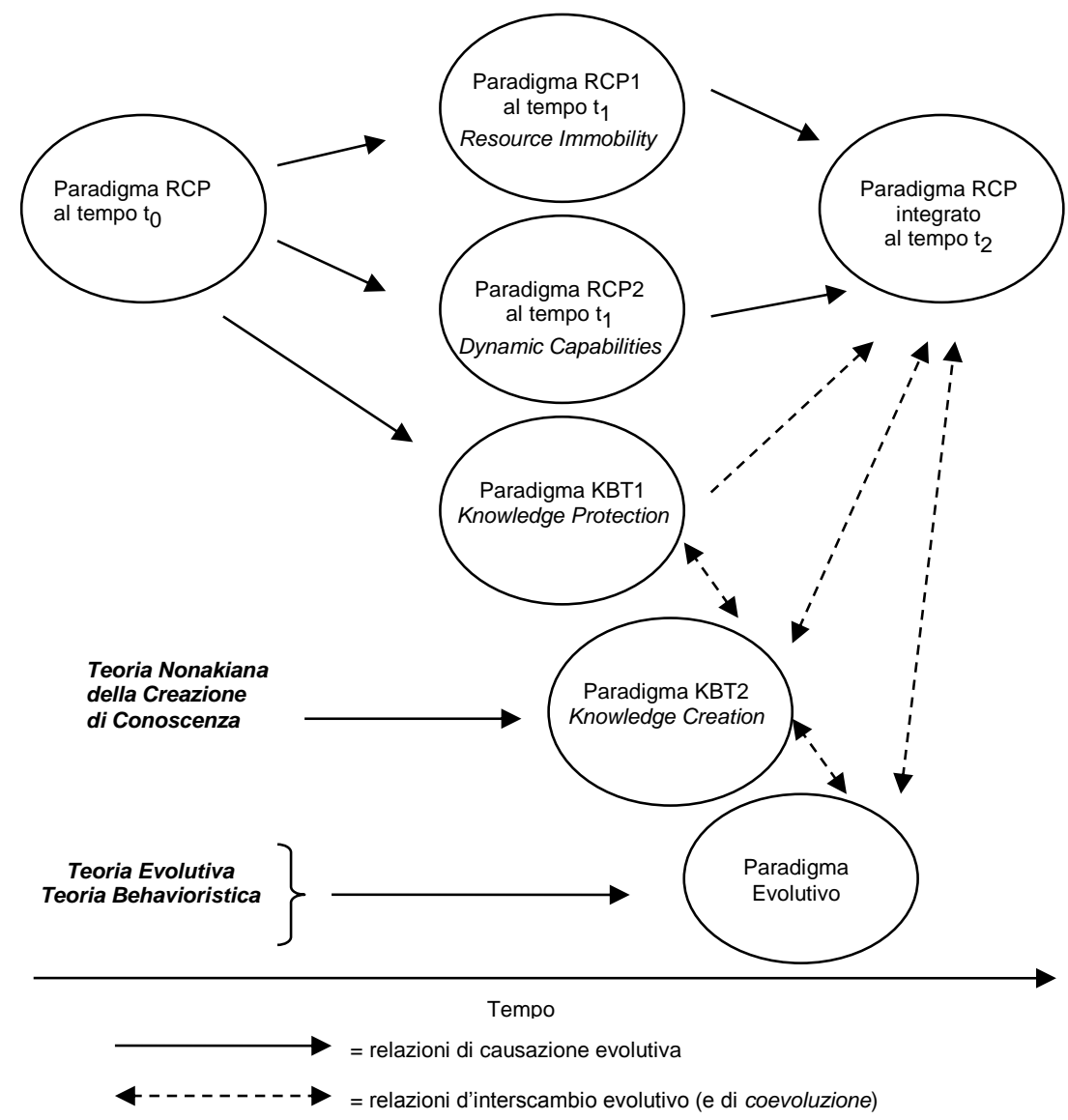

Fonte: Elaborazione a cura dell'autore 


\subsection{Paradigma evolutivo e mutamento delle fonti del vantaggio competitivo}

Grazie all'essenziale contributo della prospettiva evolutiva, si è tracciato un profilo della successione e dell'interazione dei paradigmi della strategia d'impresa e si sono poste in evidenza le fonti specifiche del vantaggio competitivo di ognuno di essi (Tabella 2). Si è messo in tal modo in rilievo che non è possibile identificare un unico paradigma in strategia d'impresa. Questo giacché il campo strategico evolutivo si presenta oggi come un campo teoreticamente ricco e variegato, che propone quattro peculiari approcci paradigmatici i quali presentano altrettante fonti del vantaggio competitivo, che contribuiscono a qualificare la parte variabile del concetto di strategia.

Fig. 4d: Dinamiche di evoluzione e di coevoluzione dei paradigmi della strategia d'impresa

(D) Coevoluzione dei paradigmi RCP e KCP

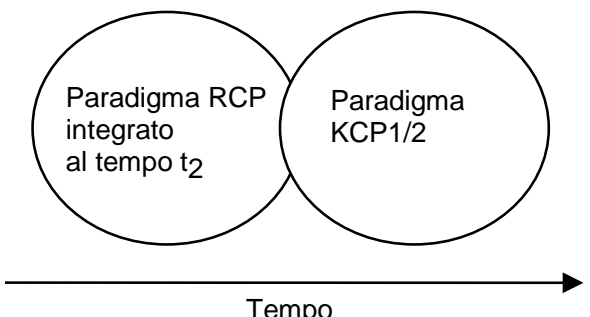

Fonte: Elaborazione a cura dell'autore

Fig. 4e: Dinamiche di evoluzione e di coevoluzione dei paradigmi della strategia d'impresa

(E) Coevoluzione dei paradigmi SCP, RCP, KCP1/2 ed evolutivo

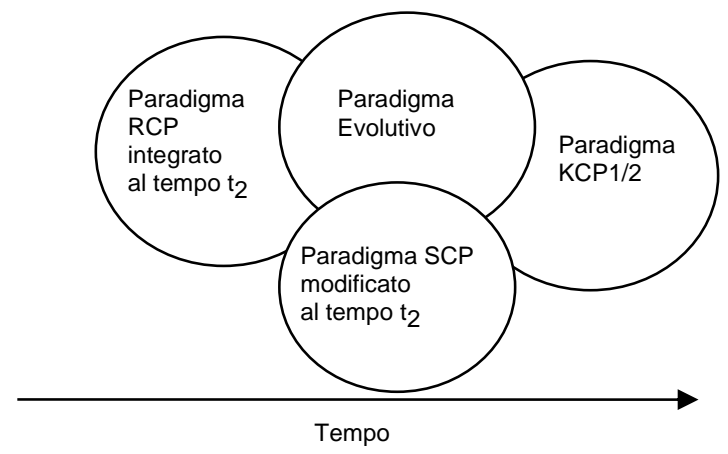

Fonte: Elaborazione a cura dell'autore 
Tab. 2: Paradigmi della strategia d'impresa e fonti del vantaggio competitivo

\begin{tabular}{|c|c|}
\hline Paradigma SCP & Industry Positioning \\
\hline Paradigma RCP & Resource, Capabilities Allocation \\
\hline Paradigma KCP & Knowledge Possession \\
\hline Paradigma Evolutivo & Routine, Innovation \& Learning \\
\hline
\end{tabular}

Fonte: Elaborazione a cura dell'autore

Tale impostazione, che prova a riordinare in modo diverso l'evoluzione della strategia d'impresa e a leggerla sotto una nuova luce, rappresenta un rilevante cambio di prospettiva. Si soggiunge che l'esame delle caratteristiche salienti del paradigma evolutivo consente di porre le basi per procedere al tracciamento di alcune linee guida del cambiamento attuale nella definizione delle fonti del vantaggio competitivo in strategia d'impresa che riguardano:

a) la crisi del primo livello di analisi strategica di base che, dalla singola impresa, via via transita - talora con inevitabili interruzioni ma inesorabilmente - ad altri livelli analitici, quali quelli relativi agli aggregati e ai sistemi di imprese (Dagnino, 2000), oggi qualificati per esempio da network, alleanze, ecosistemi e piattaforme strategiche;

b) la crisi del secondo livello di analisi strategica di base del settore industriale, che oggi molto faticosamente riesce a rappresentare le situazioni ibride, ove i confini di settore non sono agevolmente rinvenibili (blurring), e ove si verificano condizioni mutevoli di convergenza multisettoriale (per esempio ICT);

c) la crisi dell'idea tradizionale, comune almeno ai primi tre paradigmi sin qui presi in analisi (SCP, RCP, e KCP), che faceva precipuo riferimento al perseguimento delle fonti di vantaggio competitivo sostenibile. Oggi tale nozione tradizionale, soprattutto a motivo dei numerosi cambiamenti tecnologici e regolamentari, risulta spesse volte non più perseguibile mentre si fa strada l'idea speculare che procede speditamene di provare a (ri)generare più fonti (talora in concatenazione) di vantaggi competitivi temporanei o di breve durata nel tempo e nello spazio (D’Aveni et al., 2010; McGrath, 2013).

Per la loro pervasività e radicalità, le tre linee di cambiamento accennate sono potenzialmente capaci di rivoluzionare alcune basi dello strategic management che conosciamo; esse pertanto influiscono certamente sull'evoluzione del concetto di strategia, questa volta non soltanto nella parte accessoria delle fonti del vantaggio competitivo, ma altresì nella parte core della definizione di strategia, con conseguenze che non sono al momento del tutto agevolmente immaginabili.

\section{Considerazioni conclusive}

In questo articolo, si è seguita la direzione interpretativa della prospettiva evolutiva, che interseca utilmente gli afflati biologico e socio-culturali. Si è adottata tale prospettiva per discutere criticamente il concetto di strategia (in particolare le 
parti core e variabile) con riguardo ai paradigmi dominanti della strategia d'impresa.

La chiave logico-intepretativa evolutiva sembra infatti particolarmente appropriata perché, in ultima analisi, si considera il management strategico alla stregua di una "tecnologia sociale", che presenta degli andamenti nel tempo e nello spazio trattabili con l'approccio evolutivo, che è stato applicato sia all'evoluzione della tecnologia e dell'innovazione in economia generale e in economia d'impresa, sia all'evoluzione delle istituzioni.

Muovendo da tali premesse si sono discusse cinque ipotesi fra loro interrelate che conducono a concetti di strategia parzialmente differenti:

(a) il campo della strategia, lungi dal presentarsi come un blocco monolitico è al contrario un universo molteplice, articolato, frastagliato, popolato da un crogiuolo di pianeti, di astri e di costellazioni, e, proprio per questo, assai probabilmente neppure si può definire come un paradigma unico e unitario;

(b) tale universo strategico si articola in una pluralità di paradigmi, e più precisamente in quattro paradigmi ( $\mathrm{SCP}, \mathrm{RCP}, \mathrm{KCP}$ ed evolutivo), che hanno avuto in maniera diversa storie, stato di avanzamento e successo evolutivo (macroevoluzione);

(c) i paradigmi in oggetto seguono percorsi evolutivi, almeno in taluni periodi, altamente idiosincratici e presentano sia sviluppi peculiari al loro interno, sia sviluppi, ora divergenti ora convergenti, nell'interazione fra differenti paradigmi. Gli sviluppi paradigmatici possono essere talora (crono)logicamente paralleli, ma non sempre sono costanti e neppure regolari (microevoluzione) e presentano fonti del vantaggio competitivo altamente idiosincratiche;

(d) $\mathrm{i}$ percorsi di sviluppo (eso-endogeni) dei paradigmi in discorso e le loro interrelazioni possono essere interpretati utilmente con la lente biologicoevolutiva estesa o integrata dalla sensibilità socio-culturale (metaevoluzione);

(e) la storia evolutiva pluriparadigmatica ricostruita in relazione all'evoluzione del concetto di strategia è il frutto del contemporaneo affinamento delle strumentazioni epistemiche, dell'ispessimento delle fonti del vantaggio competitivo in strategia d'impresa e dell'utilizzo della lente interpretativa dell'epistemologia evolutiva.

Tutto ciò indica che la strategia è un'area di studi relativamente aperta e che il pluralismo prevalente in strategic management è sintomo di elevate ricchezza ed effervescenza intellettuale, ma costituisce al contempo un limite posto dall'assenza di sostanziale unità concettuale e di standardizzazione. Il messaggio di Thomas Kuhn (1970) in tal senso è che le discipline scientifiche possono conseguire considerevoli effetti di rete nelle fasi di scienza normale e quindi di uniparadigmaticità, giacché la standardizzazione su un'unica Weltanshaaung apporta benefici non indifferenti, quali minori costi di comunicazione e più efficace coordinamento nella divisione scientifica del lavoro. In tal prospettiva, la strategia d'impresa godrebbe di maggior efficienza qualora vi fosse un unico paradigma di riferimento comunemente accettato e un unico concetto di strategia. Il pluralismo e la pluriparadigmaticità costituiscono una sorta di condizione minima di inefficienza. 
È nondimeno utile rilevare che vi può essere unità nella diversità e che essa può mostrarsi vantaggiosa dal punto di vista evolutivo. Cosa significa "unità nella diversità"? Significa che i paradigmi della strategia, pur depositari di concezioni e portatori di messaggi per vari aspetti differenti, o talora antitetici fra di loro, mostrano tutti (con la parziale eccezione del paradigma evolutivo) una struttura causale omogenea e un'accentuata attenzione sull'efficienza e un'enfasi condivisa sulle performance. Questo comporta che, dal punto di vista epistemologico, non vi è una diversità conclusiva e non vi sono confini e barriere definitive fra paradigmi della strategia. Esistono invece le precondizioni logiche che sollecitano l'immaginazione creativa dello studioso per definire ed espandere le superfici di contatto interparadigmatiche superando i confini 'strategici' fra paradigmi. Un confine strategico è inevitabilmente un confine storicamente e fattualmente instabile e un concetto flessibile.

Si sostiene quindi che lo sviluppo del concetto di strategia può (e deve) provenire non soltanto dall'interno, ma altresì dal confronto con altre interpretazioni della strategia e prospettive delle scienze sociali. Si auspicano pertanto fertilizzazioni incrociate e analitiche fra prospettive strategiche e altre prospettive d'analisi.

\section{Bibliografia}

ANDREWS K.R. (1971), The Concept of Corporate Strategy, Homewood: Irwin.

BADEN-FULLER C., STOPFORD J (1994), Rejuvenating the Mature Business, Harvard Business School Press, Cambridge, MA.

BARNETT W.P., BURGELMANN R.A. (1996), "Evolutionary Perspectives on Strategy", Strategic Management Journal, Summer Special Issue, vol. 17, n. S1, pp. 5-19.

BARNEY J.B. (1991), "Firm Resources and Sustained Competitive Advantage", Journal of Management, vol. 17, n. 1, pp. 99-120.

BARNEY J.B., ZAJAC E. (1994), "Competitive Organizational Behavior: Toward an Organizationally-Based Theory of Competitive Advantage", Strategic Management Journal, Winter Special Issue, vol. 15, n. S1, pp. 5-9.

BAUM J.A.C., SINGH J.V. (a cura di) (1994), Evolutionary Dynamics of Organizations, Oxford University Press, New York.

BOYD R., RICHERSON P.J. (1985), Culture and the Evolutionary Process, University of Chicago Press, Chicago.

CAMPBELL D.T. (1974), "Evolutionary Epistemology", in Schlipp P.A. (a cura di), The Philosophy of Karl Popper, vol. 14 I \& II, Open Court, La Salle, IL (trad. it., Epistemologia evoluzionistica, Armando, Roma, 1981).

CERUTI M. (1985), "La hybris dell'onniscienza e la sfida della complessità", in Bocchi G.Ceruti M. (a cura di), La sfida della complessità, Feltrinelli, Milano.

CYERT R.M., MARCH J.G. (1963), A Behavioral Theory of the Firm, Prentice-Hall, Englewood Cliffs, NJ (trad. it., La teoria del comportamento d'impresa, F.Angeli, Milano).

DAGNINO G.B. (2000), Conoscenza, complessità e sistemi di imprese, Giappichelli, Torino.

DAGNINO G.B. (2005), I paradigmi dominanti negli studi di strategia d'impresa, Giappichelli, Torino. 
DAGNINO G.B. (2006), "Il paradigma evolutivo in strategia d'impresa: approccio controverso o solida prospettiva conoscitiva?", Economia e Politica Industriale, vol. 33, n. 4, pp. 103-132.

D'AVENI R.A., DAGNINO G.B., SMITH K.G. (2010). "The Age of Temporary Advantage", Strategic Management Journal, vol. 31, n. 13, pp. 1371-1385.

DURHAM W.H. (1991), Coevolution. Genes, Culture, and Human Diversity, Stanford University Press, Stanford (CA).

EISENHARDT K.M., MARTIN J.A. (2000), "Dynamic Capabilities: What are They?", Strategic Management Journal, Special Issue, vol. 21, n. 10-11, pp. 1105-1121.

EISENHARDT K.M., SANTOS F.M. (2002), "Knowledge-based view: A new theory of strategy?", in A. Pettigrew-H. Thomas-R. Whittington (a cura di), Handbook of Strategy and Management, Sage, Thousands Oak, CA.

FAULKNER D.O., CAMPBELL A. (a cura di) (2003), The Oxford Handbook of Strategy, Oxford University Press, New York.

FOSS N.J. (2003), "The Strategic Management and Transaction Cost Nexus: Past Debates, Central Questions and Future Research Possibilities", Strategic Organization, vol. 1, n. 2, pp. 139-169.

GAVETTI G., LEVINTHAL D.A., OCASIO W. (2007), "Neo-Carnegie: The School's Past, Present, and Reconstructing for the Future", Organization Science, vol. 18, n. 3, pp. 523-536.

GHEMAWAT P. (2002), "Competition and Business Strategy in Historical Perspective", Business History Review, vol. 76, n. 1, pp. 37-74.

GRANT R.M. (1996), "Toward a Knowledge-Based Theory of the Firm", Strategic Management Journal, Winter Special Issue, vol. 17, n. S2, p.109-122.

GRANT R.M. (2005), Contemporary Strategy Analysis. Concepts, Techniques, Applications, Blackwell, Oxford, $5^{\text {a }}$ ed.

HAYEK F.A. (1945), "The Use of Knowledge in Society", American Economic Review, vol. 35 , n. 4 , pp. 519-530.

HAYEK F.A. (1988), Conoscenza, mercato e pianificazione, Il Mulino, Bologna.

HITT M.A., FREEMAN R.E., HARRISON J.S. (a cura di) (2001a), The Blackwell Handbook of Strategic Management, Blackwell, Malden, MA.

HITT M.A., IRELAND R.D., HOSKISSON R.E (2001b), Strategic Management. Competitiveness and Globalization, South Western College, Cincinnati, $4^{\mathrm{a}}$ ed.

HOSKISSON R.E., HITT M.A., WAN W.P., YIU D. (1999), "Theory and Research in Strategic Management: Swings of a Pendulum”, Journal of Management, vol. 25, n. 3, pp. 417-456.

HULL D.L. (1988), Science as a Process. An Evolutionary Account of the Social and Conceptual Development of Science, University of Chicago Press, Chicago.

HULL D.L. (2001), "In Search of Epistemological Warrant", in Hayes C.-Hull D.L. (a cura di) (2001). Selection Theory and Social Construction. The Evolutionary Naturalistic Epistemology of Donald T. Campbell, State University of New York Press, Albany.

JAQUEMIN A.P., DE JONG H.W. (1977), European Industrial Organization, McMillan, London.

KUHN T.S. (1970), The Structure of Scientific Revolutions, The University of Chicago Press, Chicago.

LAUDAN L. (1978), Progress and Its Problems, University of California Press, Berkeley.

MARCH J.G., SIMON H.A (1958), Organizations, Wiley and Sons, New York.

MCGRATH R.G. (2013), The End of Competitive Advantage, Harvard Business Publishing. 
MERTON R. (2002), La serendipity nella ricerca sociale e politica: cercare una cosa e trovarne un'altra, Luiss, Roma (con S. Fallocco).

MORIN E. (1990), La Méthode IV. (trad. it., Le idee: habitat, Feltrinelli, 1993).

NELSON R.R., WINTER S.G. (1982), An Evolutionary Theory of Economic Change, Belknap Press, Cambridge, MA.

NELSON R.R., WINTER S.G. (2002), "Evolutionary Theorizing in Economics", Journal of Economic Perspectives, vol. 16, n. 2, pp. 23-46.

NONAKA I. (1994), "A Dynamic Theory of Organizational Knowledge Creation", Organization Science, vol. 5, n. 1, pp. 14-37.

NONAKA I., TOYAMA R., NAGATA A. (2000), "A Firm as a Knowledge-creating Entity: A New Perspective on the Theory of the Firm", Industrial and Corporate Change, vol. 9. n. 1, pp. 1-20.

NONAKA I., TOYAMA R. (2002). "A Firm as a Dialectical Being: Towards a Dynamic Theory of a Firm", Industrial and Corporate Change, vol. 11, n. 5, pp. 995-1009.

PETERAF M.A. (1993), "The Cornerstones of Competitive Advantage: A Resource-Based View", Strategic Management Journal, vol. 14, n. 3, pp. 179-191.

PETTIGREW A., THOMAS H., WHITTINGTON R. (a cura di) (2002), Handbook of Strategy and Management, Sage, Thousands Oak, CA.

PORTER M.E. (1980), Competitive Strategy, The Free Press, New York.

PORTER M.E. (1981), "The Contributions of Industrial Organizations to Strategic Management", Academy of Management Review, vol. 6, n. 4, pp. 609-620.

PORTER M.E., LIEBESKIND J. (1996), "Knowledge, Strategy and the Theory of the Firm", Strategic Management Journal, Wnter Special Issue, vol. 17 n. S2 pp. 93-107.

ROTHAERMEL F. (2012), Strategic Management: Concepts and Cases, McGraw-Hill, New York.

RUMELT R.P. (1991), “How Much Does Industry Matter?”, Strategic Management Journal, vol. 12, n. 3, pp. 167-185.

RUMELT R.P., SCHENDEL D.E., TEECE D.J. (a cura di) (1994), Fundamental Issues in Strategy. Cambridge, MA: Harvard University Press.

SALONER G., SHEPARD A., PODOLNY J. (2001), Strategic Management, John Wiley, Chichester.

SCHERER E.M., ROSS J. (1990), Industrial Market Structure and Economic Performance, Houghton-Mifflin, 3a ed, Boston.

SCHULZE W.S. (1994), "The Two Schools of Thought in Resouce-Based Theory: Definitions and Implications for Research", in P. Shrivastava, A. Duff e J. Dutton (a cura di), Advances in Strategic Management, vol. 10A, Jai Press, Greenwich.

SCHUMPETER J.A. (1954), History of Economic Analysis, Allen \& Unwin, Londra (trad. it., Storia dell 'analisi economica, Boringhieri, 1972).

TEECE D.J., PISANO G., SHUEN A. (1997), "Dynamic Capabilities and Strategic Management”, Strategic Management Journal, vol. 18, n. 7, pp. 509-533.

THOMPSON A.A.-STRICKLAND A.J. (2001) Strategic Management: Concepts and Cases, McGraw-Hill/Irwin, Boston, $12^{\text {th }}$ ed.

YOUNG G. (1995), "Comment: The Resource-based View of the Firm and 'Austrian' Economics. Integration to Go Beyond the S-C-P Paradigm of Industrial Organization Economics", Journal of Management Inquiry, vol. 4, n. 4, pp. 333-340. 